\title{
Téoros
}

Revue de recherche en tourisme

\section{Analyse du comportement des décideurs en matière de développement touristique régional en milieu insulaire}

\section{Denis Serra}

Volume 21, numéro 2, été 2002

URI : https://id.erudit.org/iderudit/1072408ar

DOI : https://doi.org/10.7202/1072408ar

Aller au sommaire du numéro

Éditeur(s)

Université du Québec à Montréal

ISSN

0712-8657 (imprimé)

1923-2705 (numérique)

Découvrir la revue

Citer cet article

Serra, D. (2002). Analyse du comportement des décideurs en matière de développement touristique régional en milieu insulaire. Téoros, 21(2), 56-64. https://doi.org/10.7202/1072408ar d'utilisation que vous pouvez consulter en ligne. 


\section{Analyse du comportement des décideurs en matière de développement touristique régional en milieu insulaire}

\section{Denis Serra}

e fait, pour une région touristique, d'appuyer son développement sur ses propres spécificités (forme de développement identitaire), même si elles sont moins performantes que celles de la concurrence - pour celles qui ne sont pas identitaires -, Iui permet souvent d'augmenter son efficience globale, grâce à l'augmentation de son avantage comparatif (Serra, 2001).

Il existe toutefois quelques difficultés relatives à l'organisation d'un territoire selon un tel type de raisonnement. Elles ne viendront pas forcément, comme on pourrait le craindre dans un premier temps, de la population locale, mais pourraient provenir, dans certains cas, d'une mauvaise « coordination » de l'offre, ou plus précisément des décideurs.

La spécialisation, pour être effective, nécessite évidemment une forme de coopération inter-zone (donc efficace), chaque décideur devant accepter de se spécialiser. $\mathrm{Ce}$ genre de coopération n'est pas toujours évident à mettre en œuvre, puisqu'un décideur n'est pas toujours rationnellement amené à choisir de coopérer. Il semble donc nécessaire de se pencher sur les méthodes de raisonnement des décideurs locaux afin de cerner d'éventuelles limites au bon déroulement de la spécialisation par zone (et de tenter de les pallier par la suite).

Nous étudions ici le comportement des acteurs du tourisme - professionnels du tourisme et décideurs politiques - en nous inspirant des méthodes de travail de chercheurs appartenant à une branche de l'économie qui analyse des conflits entre individus. Dans cette branche, les conflits sont des « jeux », les individus sont des « joueurs » et la théorie est la « théorie des jeux ». Nous en présentons les principaux éléments pour les appliquer ensuite au problème que pourrait poser la mise en place d'une stratégie de développement touristique basée sur la spécialisation par zone. Enfin, nous proposons divers outils qui seraient susceptibles d'aider à sortir de l'impasse à laquelle peuvent conduire certaines situations particulières.

\section{Éléments de la théorie des jeux, principes de base, définitions}

«On appelle jeu une situation dans laquelle plusieurs individus ont à prendre des décisions dont dépendent des résultats qui les concernent tous » (Attali, 1972). Les jeux de bridge, d'échecs ou de poker constituent des exemples d'une telle situation. Cependant, la définition est suffisamment générale pour couvrir des phénomènes tels les conflits militaires, les conflits de partis politiques ou la concurrence économique et même toute activité sociale où des hommes affrontent d'autres hommes. Nous pouvons donc aussi traiter le cas qui nous intéresse comme un jeu où les deux élus responsables du développement touristique de leur zone doivent faire des choix économiques dont les résultats seront liés.

Guerrien (1993) définit ainsi le jeu :

Selon l'acceptation courante, un jeu est une situation où des individus (les joueurs) sont conduits à faire des choix parmi un certain nombre d'ac- tions possibles, et dans un cadre défini à l'avance (les règles du jeu), le résultat de ces choix constituant une issue du jeu, à laquelle est associé un gain, positif ou négatif, pour chacun des participants.

Un joueur est donc un individu qui cherche à prendre des avantages dans un débat où la procédure est donnée. Ainsi, nos deux élus, suivant leurs décisions, les règles du jeu et l'environnement extérieur, seront vainqueurs (ou vaincus) l'un ou l'autre (ou l'un et l'autre). On trouve ici les fondements de la théorie de la décision selon laquelle un individu doit choisir une décision (appelée aussi tactique) en fonction de l'étendue de ses conséquences. Or, ici, chaque conséquence dépendra non seulement de sa propre décision, mais aussi de celle de son « adversaire ». Deux situations peuvent alors caractériser le jeu : la coopération ou la lutte. Cependant, les individus qui s'affrontent dans un même jeu peuvent avoir, sur certains points, des intérêts complémentaires alors qu'ils s'opposent sur d'autres.

Les choix rationnels visent l'obtention d'un gain maximal ; ils dépendent de façon décisive du cadre dans lequel ils s'exercent (règles du jeu) et de l'information dont disposent les joueurs. "Choisir une tactique revient ainsi, pour un joueur, à prendre globalement, avant de se mettre à jouer, toutes les décisions élémentaires qu'il peut être amené à prendre au cours du jeu» (Bouzitat, 1965).

On parle d'information complète si chacun des participants connait :

- son ensemble de choix,

- l'ensemble de choix des autres joueurs, 
- toute la gamme des issues possibles et les gains qui leur sont associés,

- les motifs des autres joueurs (en plus des siens propres).

Il est généralement supposé que les joueurs ont un comportement rationnel et cherchent donc à maximiser leurs gains. Le fait que chacun connaisse les motifs et les domaines de choix des autres signifie que tout joueur peut «se mettre dans la peau des autres » avant de prendre sa décision. Dans la mesure où tout le monde procède de même, il y a enchaînement sans fin : « je sais que les autres peuvent se mettre à ma place, qu'ils savent que je le sais, que je sais qu'ils savent que je le sais » et ainsi de suite. Ce jeu de miroirs ne fait que traduire la conscience qu'a chaque individu de la rationalité des autres, tout au moins lorsqu'il y a information complète. On dit, quand cette hypothèse est vérifiée, qu'il y a « connaissance commune de la structure du jeu », de la part de tous ceux qui y participent (Guerrien, 1993). Le jeu auquel participent nos décideurs est un jeu à information complète.

Information parfaite : quand il y a information complète, chaque joueur connaît toutes les données du problème (possibilités de choix, issues, gains, motifs), pour lui et pour les autres. Toutefois, pour qu'un jeu soit totalement défini, il faut que ses règles précisent l'ordre des coups; trois types de situations peuvent alors être envisagés : soit les joueurs font leurs choix de façon séquentielle, dans un ordre précis fixé à l'avance; soit ils prennent leurs décisions simultanément ; soit ils sont face à des situations « mixtes », avec des coups successifs et des coups simultanés. Évidemment, les choix des joueurs et la solution du jeu (si elle existe) varieront généralement en fonction du type de situation étudié.

Pour le cas qui nous intéresse, nous admettrons que les décisions sont prises simultanément puisque le développement de la région nécessite le développement de chacune de ses zones de manière instantanée. Les décisions seront alors prises dans le cadre d'un jeu à information imparfaite, du fait de l'incertitude inhérente aux choix simultanés.

\section{Premier jeu}

Les joueurs de notre jeu sont des décideurs (les maires et les autres élus ou les responsables du développement régional de la commune). Les décisions qu'ils prendront sont relatives à l'implantation d'établissements d'hébergement touristique sur leur propre zone. Pour simplifier le jeu au maximum (et privilégier le raisonnement), nous considérons que chaque décideur n'a en fait le choix qu'entre deux types d'hébergement : l'hôtel (noté $\mathrm{H}$ ) et le camping (noté $\mathrm{C}$ ). Par ailleurs, nous ne différencions que deux catégories de décideurs, en fonction des caractéristiques de leur zone de décision, c'est-à-dire de la zone sur laquelle ils devront implanter l'établissement de leur choix. Ainsi, nous distinguons ceux qui occupent une zone dite « luxe $e^{1}$ » de ceux qui occupent une zone que nous qualifions de « verte ${ }^{2} »$. Nos joueurs sont donc notés respectivement «DL » et «DV ».

\section{Les règles du jeu}

Dans le but de faciliter la compréhension du jeu, nous posons les règles suivantes :

- Seuls deux décideurs s'opposent à chaque jeu.

- Les joueurs opposés occupent des zones voisines, formant ainsi une macro-zone.

- Chaque joueur dispose de subventions qui lui permettent de construire trois établissements de son choix ; il choisira toujours d'utiliser toutes les subventions qui sont à sa disposition. Le jeu consiste donc pour lui en la détermination de la combinaison des différents types d'établissements (hôtel : H, ou camping : C) qui lui procurera la meilleure utilité en termes de flux monétaire 3 à la sortie du jeu. Il lui faudra donc maximiser ce flux monétaire.

- Les «contraintes » sont, en fait, les éléments qui font partie de l'environnement du joueur et qui interviendront dans le calcul de son gain. Le joueur n'a aucun contrôle sur ces éléments, mais il les connaît; il sait que le joueur qui lui est opposé les connait et qu'il sait que lui aussi les connaît, cela à l'infini. Les contraintes peuvent être exposées comme suit :
- Un hôtel engendre un flux monétaire supérieur à celui engendré par un camping, quelle que soit la nature de la zone sur laquelle on l'implante (luxe ou verte). L'écart se fait principalement sur le tarif de la nuit d'hébergement et sur le fait que « généralement » la clientèle qui préfère l'hôtel au camping dépense davantage dans la zone.

- Un établissement, quel qu'il soit (hôtel ou camping), engendre un flux monétaire plus important dans une zone de luxe que dans une zone verte, essentiellement du fait que la zone de luxe dispose (par définition) de plus de commerces que la zone verte.

- Cependant, l'écart est moindre en ce qui concerne les campings (comparé à l'écart existant entre les flux monétaires d'un hôtel selon sa position en zone de luxe ou verte). Il semble en effet raisonnable de penser que la clientèle d'un hôtel en zone verte dépense tout de même plus que celle d'un camping (même) en zone de luxe. Toutefois, cette supposition demeurant peut-être discutable pour d'aucuns, nous choisissons (pour limiter le risque d'erreur lié à ce raisonnement) d'accorder un flux monétaire (moyen) équivalent aux deux cas de figure en question.

- Enfin, il faut tenir compte de la demande. La demande en campings reste environ deux fois plus importante que la demande en hôtels. (Cela dépend évidemment du lieu de villégiature, mais cette recherche étant tirée d'une étude réalisée en Corse [Serra 1997], nous considérons cette condition générale comme une contrainte... le but de cet article demeurant l'étude du comportement et la méthode de raisonnement des décideurs.)

- Les décisions sont prises de façon simultanée.

Le tableau 1 résume les contraintes et permet d'illustrer plus facilement les développements à venir de notre étude.

Nous opposerons, pour la première confrontation, deux décideurs qui occupent un même 
TABLEAU 1

Flux monéraire (en unités monétaires - U.M.) engendrés par les hôtels el les campings, selon la zone (luxe ou verle)

\begin{tabular}{|l|c|c|}
\hline Flux monétaire & Zone de luxe & Zone verte \\
\hline Hôtel & 500 & 200 \\
\hline Camping & 200 & 150 \\
\hline
\end{tabular}

type de zone, par exemple deux zones de luxe constituant la macro-zone (L1-L2). Nous pouvons imaginer qu'il s'agit en fait d'une même zone (mêmes critères), mais étalée sur deux communes et donc gérée par deux décideurs distincts (nous considérons dans notre modèle que les décisions sont prises par le maire). Le premier joueur est donc noté DL1 et le second DL2.

La demande globale pour la macro-zone est de deux hôtels et de quatre campings, soit un hôtel et deux campings par zone. La demande est la même pour les deux sites puisqu'ils sont identiques ; cette particularité signifie donc qu'il n'y aura de « report » de la demande d'un site sur l'autre que si le premier ne peut y répondre correctement. En effet, les sites L1 et L2 étant identiques, il n'y a aucune raison pour que la clientèle du premier soit détournée sur le second. Ainsi, si L1 dispose d'un hôtel et L2 de deux, seul l'un de ces deux derniers sera utilisé puisque la demande globale est de $2 \mathrm{H}$ et que la moitié sera « utilisée » par L1. Par contre, si L1 choisit de ne pas implanter d'hôtel, la demande qu'il ne peut satisfaire sera intégralement reportée sur L2, puisque ce site apporte aux visiteurs exactement la même satisfaction que le premier en raison de la similarité de leurs caractéristiques.

\section{Les différentes stratégies}

Chaque joueur aura à choisir entre les quatre stratégies qui s'offrent à lui :

- Stratégie A :

implanter 1 hôtel +2 campings

- Stratégie B :

implanter 2 hôtels +1 camping

- Stratégie C :

implanter 3 campings

- Stratégie D :

implanter 3 hôtels
- A/A : (respectivement DL1 joue A et DL2 joue $A$ ) les deux joueurs ont un gain de $1 * 500+2 * 200=900$ U.M. (noté 9 dans le tableau de la forme stratégique). La demande par zone est respectée (et donc celle de la macro-zone).

- B/A : (DL1 joue B et DL2 joue A) DL1 ne remplit qu'un de ses deux hôtels et son camping (d'après la demande) soit $500+200=700$ (gain noté 7), tandis que DL2 remplit son hôtel, ses deux campings et bénéficie du report d'au moins une partie de la demande en camping non satisfaite par DL1 (on l'estime équivalente à un flux monétaire de 100 U.M.), soit : $500+2 * 200+100=1000$ (ce gain est noté 10 dans le tableau de la forme stratégique).

- C/A : DL1 remplit deux de ses trois campings (gain noté 4), DL2 obtient $500+2 * 200+100=1000$ (noté 10 ). Cette fois c'est le report de la demande en hôtel non satisfaite en L1 qui permet à DL2 d'obtenir 100 U.M. supplémentaires.

- D/A : DL1 obtient 500 grâce à l'un de ses trois hôtels (soit un gain noté 5). DL2 gagne $500+2 * 200+100$ (noté 10 ).

- On comprend que les gains seront les mêmes si les joueurs inversent symétriquement leurs stratégies. Il est donc inutile de détailler $\mathrm{A} / \mathrm{B}, \mathrm{A} / \mathrm{C}$ et $\mathrm{A} / \mathrm{D}$, respectivement équivalentes à $\mathrm{B} / \mathrm{A}, \mathrm{C} / \mathrm{A}$ et $\mathrm{D} / \mathrm{A}$.

- B/B : les deux joueurs remplissent un de leurs hôtels (ne bénéficient d'aucun report issu d'hôtels en trop grand nombre sur chaque zone) et leur camping et obtiennent chacun 100 U.M. en raison du manque de campings sur la macro-zone. Soit pour chacun un gain égal à $500+200+100=800$ (noté 8 dans le tableau).
- C/B : DL1 gagne $2 * 200+100$ (noté 5) correspondant à ses deux campings et au report de la demande du camping manquant en L2 ; DL2 gagne $2 * 500+200$ (noté 12 ) $\{$ Idem pour B/C $\}$.

- D/B : Gain pour DL1 : 1*500 (noté 5), il ne remplit qu'un hôtel. Gain pour DL2 : $1 * 500+200+100$ (noté 8 ) correspondant à un seul de ses hôtels, son camping et un report de 100 U.M. en raison du manque de camping sur L1 $\{$ Idem pour $\mathrm{B} / \mathrm{D}\}$.

- C/C : $2 * 200$ pour DL1 et DL2 (noté 4), puisqu'ils remplissent chacun deux de leurs campings (la demande sur la macrozone est de quatre campings en tout).

- D/C : DL1 remplit ses deux hôtels $500 * 2=1000$ (noté 10) et DL2 deux de ses campings plus un report des campings manquants en L1, soit $2 * 200+100=500$ (noté 5 ) $\{$ Idem pour C/D $\}$.

- D/D : Chaque joueur obtient, par l'occupation de l'un de ses trois hôtels, un gain égal à 500 U.M. (noté 5).

En théorie des jeux, on dit qu'il s'agit là d'un jeu non coopératif ; il n'y a pas d'accord préalable entre les joueurs. Nous avons donc un jeu à information complète, mais imparfaite. La représentation généralement utilisée en théorie des jeux pour analyser le déroulement du jeu, la méthode de raisonnement des participants, est appelée forme stratégique ou normale. La forme stratégique fait appel à un (ou des) tableau(x) de chiffres donnant les gains des joueurs associés à chacune des issues possibles du jeu ; les lignes et les colonnes correspondent aux diverses stratégies.

Notre jeu prend donc la forme stratégique suivante :

\begin{tabular}{|c|c|c|c|c|c|c|c|c|}
\hline$D\lfloor 2 \downarrow / D L I \rightarrow$ & $A$ & & B & & $c$ & & 0 & \\
\hline$A \rightarrow$ & 9 & 9 & 7 & 10 & 4 & 10 & 5 & 10 \\
\hline$B \rightarrow$ & 10 & 7 & 8 & 8 & 5 & 12 & 5 & 8 \\
\hline$C \rightarrow$ & 10 & 4 & 12 & 5 & 4 & 4 & 10 & 5 \\
\hline$D \rightarrow$ & 10 & 5 & 8 & 5 & 5 & 10 & 5 & 5 \\
\hline
\end{tabular}




\section{Concept de solution}

La prise de décision est fondée sur le principe de rationalité du décideur, rationalité qui le pousse à maximiser son profit en tenant compte de toutes les issues possibles du jeu et donc aussi de la rationalité de son adversaire qui poursuit le même but. La première démarche du décideur est alors d'éliminer les stratégies dominées, c'est-àdire celles qui, dans tous les cas (quelle que soit la stratégie choisie par l'autre), lui apporteront des gains inférieurs à ceux engendrés par au moins une autre stratégie. Dans un deuxième temps, le joueur élimine également de lui-même les stratégies dominées de son adversaire qu'il sait rationnel (il sait que son adversaire suit le même raisonnement que lui et donc qu'il ne jouera en aucun cas une stratégie dominée). La solution par élimination des stratégies dominées demande de la part de chaque joueur d'effectuer une succession de calculs en se mettant « dans la peau de l'autre », donc en anticipant (correctement) son comportement, ce qui n'est possible que s'il y a connaissance commune de toutes les caractéristiques du jeu. Évidemment, plus le nombre de stratégies et de joueurs est grand, plus ces calculs peuvent devenir complexes.

Selon le même principe, nos décideurs raisonneront comme suit :

- DL1 constate que s’il choisit la stratégie A, il aura un gain de :

- 9 si DL2 joue $A$,

- 10 si DL2 joue B,

- 10 si DL2 joue $C$,

- 10 si DL2 joue D.

On dira que la matrice des gains associée à la stratégie $\mathrm{A}$ est, pour le joueur 1 : $(9,10,10,10)$. De la même façon, DL1 constate que les matrices des gains associées aux stratégies $B, C$ et D sont pour lui respectivement : $(7,8,12,8),(4,5,4,5)$ et $(5,5,10,5)$.

Le décideur DL1 alors remarque que la matrice des gains associée à la stratégie A est en tous points supérieure à celle associée à la stratégie $\mathrm{C}:(9,10,10,10)>(4,5,4,5)$. Cela signifie que s'il joue A, son gain sera plus important que s'il joue $\mathrm{C}$, quelle que soit la stratégie adoptée par son vis-à-vis DL2. La stratégie $\mathrm{C}$ est donc dominée au sens de Pareto (ou Pareto-dominée) (Guerrien, 1993) par la stratégie A. Le même raisonnement l'amène à éliminer la stratégie $\mathrm{D}$ qui, même si elle n'est pas strictement dominée par A, ne lui rapportera dans le meilleur des cas (si DL2 joue C) qu'un gain équivalent à celui qu'apporterait $\mathrm{A}$ : $(9,10,10,10) \geq(5,5,10,5)$. Il reste donc au joueur les stratégies A et B que l'on qualifiera d'efficaces car « non-Pareto-dominées ».

Le joueur DL2 procède au même raisonnement qui l'amène aux mêmes conclusions, puisque les matrices des gains sont les mêmes pour les deux joueurs (occupant le même type de site).

DL1 sait que DL2 va tenir un tel raisonnement; il ne tiendra donc pas compte des gains que peuvent lui apporter ses stratégies $\mathrm{A}$ et $\mathrm{B}$ dans le cas où DL2 jouerait $\mathrm{C}$ ou D, puisqu'il n'y a rationnellement aucune chance pour que cela arrive.

Le jeu deviendra alors un jeu «tronqué » ne comprenant plus pour chaque joueur que les stratégies Pareto efficaces A et B.

\begin{tabular}{|c|c|c|}
\hline$D L 2 \downarrow / D L I \rightarrow$ & A & $B$ \\
\hline $\mathrm{A} \rightarrow$ & (9) & $\begin{array}{l}7 \\
\quad[10]\end{array}$ \\
\hline $\mathrm{B} \rightarrow$ & (10) & 8 \\
\hline
\end{tabular}

Poursuivant leur logique d'éliminations successives des stratégies dominées, dans ce nouveau jeu, chaque joueur constate que sa stratégie $B$ est dominée par la stratégie $A$, puisque : $(9,10)>(7,8)$; alors, les deux joueurs joueront $A$. On peut dire que l'issue $\mathrm{A} / \mathrm{A}$ constitue un équilibre Pareto efficace, car la solution obtenue rationnellement est la plus efficiente.

On note en effet que dans un jeu opposant deux décideurs de zone de luxe, l'équilibre obtenu « naturellement » grâce à leur rationalité correspond à l'issue qui permet le meilleur flux monétaire global. Dans l'exemple : $9+9=18$, soit un flux monétaire global de 1800 unités monétaires pour la macro-zone L1-L2. Dans un tel cas, la forme de coopération entre les décideurs qui veut qu'ils se partagent équitablement les établissements à implanter est rationnellement induite.

La stratégie de spécialisation (Serra, 2001) n'a pas d'effet visible sur deux zones similaires et ne pose donc pas de problème de décision particulier aux décideurs. Il n'en sera pas toujours ainsi.

\section{Second jeu}

\section{Nouvelle donne}

Le problème devient quelque peu plus complexe quand on oppose deux décideurs gérant l'un une zone de luxe, que nous notons DL, et l'autre une zone verte, que nous notons DV.

Nous avons vu que les différents types d'hébergement n'apportaient pas les mêmes flux monétaires dans une zone de luxe que dans une zone verte. Par ailleurs, les reports ne seront pas aussi simples à établir que dans le cas précédent puisque, par exemple, la demande en camping dans le site vert (V) est liée à la fois au site et au type d'hébergement ; si le site en question ne parvient pas à contenter la demande, la clientèle qui aura choisi l'endroit pour camper se reportera sans doute dans un camping du site voi$\sin \mathrm{L}$, mais la clientèle qui avait choisi l'endroit parce qu'il est « vert » se reportera probablement plus loin, mais toujours sur une autre zone verte.

Un problème fondamental se pose également quand la défense par chacun de son intérêt personnel a des conséquences néfastes pour tous. Le « dilemme du prisonnier » (Guerrien, 1993) est une représentation classique d'une telle situation en théorie des jeux. Dans le dilemme du prisonnier, deux joueurs sont en présence et chacun a deux options : soit coopérer, soit faire « cavalier seul ». Chacun doit choisir sans connaître la décision de l'autre. Quoi que fasse l'autre, il est plus payant de faire cavalier seul que de coopérer. Le dilemme du prisonnier consiste en ceci : si les deux joueurs font cavalier seul, ils « s'en tirent » moins bien que s'ils avaient coopéré. Contrairement à la théorie de « la main 
invisible » d'Adam Smith (1776), selon laquelle la somme des intérêts individuels conduit à l'intérêt général, ici la rationalité individuelle (visant toujours à maximiser le profit individuel), mène au pire résultat possible pour les deux joueurs, d'où le dilemme. Jean Gabszewicz (1994) donne un exemple concret de ce phénomène en mettant en scène deux vendeurs qui se disputent un marché.

Dans le cas qui nous intéresse, nous pourrions présenter les choses de la façon suivante : nous l'avons vu, la zone de luxe détient « un avantage absolu » sur la zone verte, qu'il s'agisse d'hôtels ou de campings, mais la zone verte dispose « d'un avantage comparatif $»$ dans l'exploitation de campings. Dans un tel cas, une spécialisation par zone apporterait un gain maximal aux deux joueurs. Cependant, si un seul d'entre eux optait pour la spécialisation (coopérait en n'implantant par exemple que des campings), l'autre joueur, choisissant de ne pas coopérer (en se diversifiant), récupérerait la demande en hôtel non pourvue par son vis-à-vis sans lui retourner en échange la demande en camping qu'il n'aurait pas dû satisfaire (s'il avait coopéré). Chacun tentera donc de gagner davantage en dupant « l'adversaire » (Guerrien, 1993, présente un semblable cas).

Ici :

- Si les deux décideurs coopèrent et se spécialisent, DL implantant deux hôtels obtiendra un gain de $2 * 500=1000$ (noté 10 ) et DV avec quatre campings obtiendra $4 * 150=600$ (noté 6 ). La demande de la macro-zone V-L est respectée.

- S'ils ne coopèrent pas (tous deux implantent un hôtel et deux campings), DL obtiendra un gain égal à $500+2 * 200=900$ (noté 9) et DV obtiendra $200+2 * 150=500$ (noté 5 ).

- Si seul DL coopère ( $2 \mathrm{H}+0 \mathrm{C})$, DV construisant un hôtel et deux campings gagnera, outre les flux monétaires associés à sa clientèle naturelle (issue de la demande sur sa zone) $200+2 * 150$, le report des deux campings manquants en $\mathrm{L}$ : $2 * 100$, soit un gain total de 700 U.M. (noté 7). Tandis que DL ne remplira qu'un seul de ses hôtels puisqu'il ne bé- néficiera pas du report de $\mathrm{V}$ (puisque celui-ci contente sa demande en hôtel), soit un gain égal à 500 U.M. (noté 5).

- Enfin, si c'est DV qui coopère et lui seul (en implantant uniquement quatre campings), DL $(2 \mathrm{H}+1 \mathrm{C})$ gagnera $2 * 500+200=1200$ U.M. (noté 12), son deuxième hôtel sera rempli par la clientèle non contentée en $\mathrm{V}$, tandis que DV ne remplissant que trois de ses campings n'obtiendra que $3 * 150=450$ U.M. (noté $4,5)$.

Représentation de la forme stratégique des résultats :

\begin{tabular}{|c|c|c|}
\hline$D V \downarrow / D L \rightarrow$ & Coopère & $\begin{array}{c}\text { Ne coopère } \\
\text { pas }\end{array}$ \\
\hline Coopère $\rightarrow$ & 10 & (12) \\
\hline $\begin{array}{r}\text { Ne coopère } \\
\text { pas } \rightarrow\end{array}$ & [7] & (9) \\
\hline
\end{tabular}

\section{« Résolution 》}

Pour le joueur DL, la matrice des gains associée à la stratégie « coopérer » est dominée par celle associée à la stratégie « ne pas coopérer » $(12,9)>(10,5)$. Il en est de même pour DV puisque $(7,5)>(6,4,5)$. C'est pourquoi, rationnellement, tous deux choisiront de ne pas coopérer ; on dira qu'à cette combinaison de stratégie est associé le vecteur de gain $(5,9)$ correspondant aux gains respectifs de DV et DL.

Pourtant, la solution issue de ce choix n'est pas la plus efficiente ; on remarque effectivement que si les deux joueurs avaient coopéré, ils auraient obtenu (10) et (6) (respectivement pour DL et DV), au lieu de (9) et (5).

Dans le dilemme du prisonnier, la solution la plus efficiente n'est jamais atteinte : $(10 ; 6)>(9 ; 5)$.

On parle néanmoins d'une solution d'équilibre dans le sens défini par le mathématicien John Nash en 1950. La notion d'équilibre désigne alors une situation où chacun maximise ses gains, compte tenu du choix des autres. Un « équilibre de Nash » (Guerrien, 1993) est une combinaison de stratégies - une par joueur - telle que per- sonne n'aurait pu augmenter strictement son gain en retenant une stratégie différente de celle que lui attribue cette combinaison, compte tenu des stratégies des autres joueurs qui y figurent, ce que Guerrien résume de façon un peu vague (selon ses termes) en disant qu'un équilibre de Nash est une situation de «non-regret » : il y a équilibre de Nash si chaque joueur ne regrette pas le choix qu'il a effectué après avoir constaté celui des autres. Cette dernière définition présente l'avantage de n'entretenir aucun doute du caractère unique, irréversible et simultané des choix des joueurs. Ainsi, c'est après que chacun ait annoncé son choix que l'on peut dire s'il y a équilibre ou pas.

Dans notre second jeu, le couple de stratégies (DV ne coopère pas ; DL ne coopère pas) auquel correspond le vecteur de gain $(5,9)$ est un équilibre de Nash, puisque DV n'aurait pas gagné à coopérer (si DL s'en tient à ne pas le faire) et il en est de même pour DL. Quand l'élimination des stratégies dominées désigne une solution unique, celle-ci est forcément un équilibre de Nash.

On comprend alors dans un tel cas la difficulté que peut représenter la mise en œuvre d'une stratégie de développement touristique basée sur la spécialisation par zone, du fait qu'elle n'est réellement efficace que si elle est adoptée sur tout le territoire et donc par tous les décideurs. Quelques auteurs intéressés à la théorie des jeux ont tenté d'apporter des solutions (de vraies solutions cette fois) au dilemme du prisonnier, c'est-à-dire des moyens de sortir de l'impasse d'une solution ou d'un équilibre inefficient. Il nous faut donc à présent tenter de trouver, à notre tour et avec l'aide de leurs travaux, « le meilleur moyen » pouvant être adapté à notre problème et permettant de sortir du dilemme.

\section{Sortir du dilemme}

La sous-optimalité de la «solution » fatalement issue de la prise de décision rationnelle de nos joueurs est flagrante, les décideurs en sont conscients (puisque rationnels) ; pourtant, elle est inéluctable. On pourrait alors se poser la question de savoir 
ce que l'on entend réellement par « rationalité ». Plus clairement, dans le second jeu opposant DL et DV, force est de constater qu'il serait sinon plus rationnel de la part des décideurs, en tout cas plus « intelligent », d'avoir un comportement à première vue « irrationnel » qui consisterait à choisir la combinaison de stratégies correspondant à un gain optimal pour chacun d'eux. Il suffirait qu'il y ait une forme d'accord tacite, une négociation préalable, une entente entre les joueurs qui les conduirait à retenir l'issue leur procurant à chacun un gain supérieur à celui de l'équilibre (dit rationnel). Seulement voilà, comment être sûr que l'accord sera respecté au moment du choix, alors que la tentation sera grande, pour chacun, de « dévier» dans le but de gagner davantage ?

On propose, en théorie des jeux, de favoriser le choix de coopérer par un système de sanctions, imposé de l'extérieur et venant donc modifier les données initiales du jeu. Nous verrons dans un premier temps plus en détail cette forme «d'obligation » à coopérer sous peine de sanction ; nous en soulignerons les limites avant de présenter d'autres méthodes plus douces « d'incitation » à la spécialisation.

\section{Imposer une stratégie}

On pourrait effectivement imaginer qu'une instance hiérarchiquement supérieure à la commune rende obligatoire la spécialisation en ordonnant purement et simplement un style de développement précis à chaque décideur (selon la zone qu'il « gère »). Toutefois, cela transformerait totalement le jeu puisque les décideurs, se voyant imposer une stratégie, ne seraient plus réellement les joueurs. Cette procédure irait donc totalement à l'encontre de la volonté des instances aussi bien nationales qu'internationales de décentraliser au maximum le pouvoir concernant ce genre de décisions liées à l'aménagement du territoire (en France). C'est d'ailleurs la prise en considération de ce phénomène qui nous a poussé à créer un modèle qui fait intervenir les professionnels et les politiques au niveau le plus local possible (dans nos exemples, ce sont les maires qui gèrent).
Par ailleurs, on constate que toute forme d'obligation est souvent accueillie avec beaucoup de réticence par les individus concernés (et la population locale), même si la décision leur est favorable. Or, l'adhésion de tous vis-à-vis du projet mis en place est indispensable à un développement basé essentiellement sur le tourisme. Soulignons également que 1'une des particularités liées à l'insularité est de provoquer au sein de la population un certain besoin, sinon d'une « dose » d'autonomie en tout cas «d'autodétermination », et ce essentiellement quand il s'agit du devenir de l'île aussi bien sur le plan économique qu'organisationnel.

\section{La crainte de représailles}

Heureusement, les nombreuses recherches effectuées dans le cadre de la théorie des jeux montrent que souvent les formes d'obligation de coopérer peuvent provenir des joueurs eux-mêmes; le cas est très répandu dès qu'il s'agit d'un jeu « répété ». La vie économique et sociale ayant indéniablement un caractère répétitif, échanges, production et, plus généralement, interactions, se reproduisant dans des conditions semblables, ou presque, pendant de longues périodes, on voit régulièrement apparaître des phénomènes particuliers liés tantôt à la réputation, aux menaces de représailles ou encore à diverses formes de normes ou de conventions.

Pour le cas qui nous préoccupe, nous pouvons bien évidemment décider d'admettre que la situation menant le maire à prendre une décision quant à l'utilisation des subventions obtenues pour l'année en cour se reproduira l'année suivante, et ceci indéfiniment ; cela fait de notre jeu un jeu répété auquel s'appliqueront les phénomènes en question.

La principale conséquence qui découle de cette précision tient du fait qu'alors le joueur devra intégrer dans son raisonnement l'idée que sa façon de jouer aura des conséquences sur le « coup suivant», puisqu'elle aura donné une indication à son adversaire, et inversement, au coup suivant, il devra tenir compte du choix qu'a effectué l'autre joueur la fois précédente. Tout cela devra bien sûr être pris en compte dès le premier coup puisqu'il aura une influence sur les coups suivants et donc sur le gain total final. « Le superjeu étant forcément à plusieurs coups, les stratégies des joueurs y sont formées par des successions d'actions (une par coup) ; ces actions sont, à chaque coup, conditionnelles puisqu'elles doivent tenir compte des choix effectifs ou éventuels des autres joueurs aux coups précédents » (Guerrien, 1993).

Sachant cela, les joueurs peuvent alors adopter différents comportements visant toujours à tenter de maximiser leurs gains sans pour autant prendre trop de risques. Selon Axelrod (1984), la méthode du « donnant-donnant » est l'une des manières de jouer des plus efficientes ; elle permet de se sortir d'un équilibre sous-optimal du type dilemme du prisonnier. Elle consiste, pour un joueur, à commencer le jeu en coopérant (donc dès le premier coup), puis à imiter à chaque coup suivant la stratégie employée par l'adversaire. Ainsi, si l'autre n'a pas coopéré, il ne coopérera pas au coup suivant en guise de représailles. Si, dans le cas du dilemme du prisonnier, les « promesses » de représailles n'ont pas de prise sur l'adversaire qui sait très bien que le jeu sera de toute manière terminé à l'issue de son choix, quand le jeu se répète, elles deviennent crédibles et font donc réfléchir le second joueur. Évidemment, si nos deux décideurs choisissent cette tactique, la spécialisation se fera sans problème puisque n'ayant pas de raison de dévier tant que l'autre ne le fait pas, aucun d'eux n'aura jamais à choisir de ne pas coopérer.

Cette méthode de jeu est rationnellement réalisable puisque la répétition du jeu rend, sinon le risque encouru au premier coup moins important, en tous cas les éventuelles pertes encourues moins lourdes. En effet, dans le cadre de notre second jeu, si le décideur DL choisit (de manière irrationnelle) de coopérer, il risque de n'obtenir qu'un gain de (5) (si l'autre ne fait pas de même), alors qu'il pouvait, en faisant l'autre choix, s'assurer un gain de (9) (quoi que joue DV); il encourt donc une perte de 400 U.M., soit $(4 / 9) * 100=44 \%$ de ce qu'il pourrait obtenir à coup sûr. 


\begin{tabular}{|l|c|c|}
\hline DV $\downarrow ; \mathrm{DL} \rightarrow$ & Coopère & $\begin{array}{c}\text { Ne coopère } \\
\text { pas }\end{array}$ \\
\hline Coopère & $(6 ; 10)$ & $(4,5 ; 12)$ \\
\hline Ne coopère pas & $(7 ; 5)$ & $(5 ; 9)$ \\
\hline
\end{tabular}

Maintenant, si le jeu doit se répéter ne serait-ce que cinq fois, et si l'autre ne choisit jamais de se spécialiser, DL obtiendra en coopérant au premier coup et jamais plus après : $(5+9+9+9+9)=(41)$ au lieu des $(5 * 9)=45$ réalisables à coup sûr, soit une perte de $(4 / 45) * 100=8,8 \%$ de ce qu'il pouvait obtenir sans risque. Bien sûr, la perte est toujours de 400 U.M., mais risquer $44 \%$ de pertes pour obtenir 10 plutôt que 9 (ce qui revient à tenter une augmentation de $11,1 \%$ de ses gains) n'est pas la même chose que de risquer $8,8 \%$ de pertes toujours pour tenter l'augmentation de $11,1 \%$ (50 au lieu 45). Le risque, s'il est mathématiquement le même puisqu'il ne repose que sur le premier coup, n'a pas la même portée, pour les mêmes effets possibles.

Toutefois, cette méthode du donnant-donnant n'est « rationnellement applicable » que si le jeu se répète un nombre infini de fois. En effet, même s'il est répété un très grand nombre de fois, le dilemme du prisonnier n'admet qu'une seule issue d'équilibre : les deux joueurs ne coopèrent jamais, alors que tous deux gagneraient bien plus s'ils le faisaient.

\section{Explication}

Dans notre exemple, les décideurs sont des maires ; or, ceux-ci ne sont pas élus définitivement. Comme chacun le sait un maire, en France, n'est élu que pour six ans et cela risque fort de fausser (dans un premier temps) l'issue du sixième jeu. La dernière année, dans l'espoir d'être réélu, le joueur ne sera-t-il pas tenté de duper l'adversaire pour obtenir de meilleurs résultats dans sa commune (aucune menace de représailles ne pouvant plus l'en empêcher à ce moment là) ? La question est grande, car ce doute aura des conséquences (néfastes) sur les coups précédents : le joueur craignant d'être dupé sur le dernier coup va tenter d'être le premier opportuniste en refusant la coopération dès l'avant-dernier

coup ; mais l'autre, prévoyant une telle anticipation, choisira de ne pas coopérer au quatrième coup (pour un jeu établi sur six ans) et on remontera ainsi jusqu'au tout premier jeu où l'un et l'autre choisiront de faire cavalier seul en « représailles anticipées » sur ce que fera vraisemblablement l'autre l'année suivante.

Cette méthode de raisonnement est appelée « récurrence à rebours » et ce phénomène bien particulier est connu en théorie des jeux sous le nom du « centipède $»$ ou «paradoxe du mille-pattes de Rosenthal » (Guerrien, 1993) :

\begin{tabular}{|l|l|l|l|l|l|l|l|l|}
\hline Gain A & 1 & 0 & $\ldots \ldots-\ldots$ & 998 & 997 & 999 & 998 & 1000 \\
\hline Gain B & 1 & 3 & $\ldots \ldots-\ldots$ & 998 & 1000 & 999 & 1001 & 1000 \\
\hline
\end{tabular}

A et B peuvent stopper le jeu quand ils le désirent. Il est évident que B souhaitera arrêter à l'avant-dernier coup ; A le sait et préférera donc stopper à 999 , d'où l'intérêt pour B d'en rester à quatre coups de la fin du jeu avec 1000 , etc. La solution selon le principe de récurrence à rebours consiste à ce que A décide d'arrêter au premier coup, de sorte que les deux joueurs obtiennent un gain de 1, alors qu'ils auraient pu avoir 1000 chacun s'ils avaient décidé de continuer à chaque coup.

Les solutions fondées sur des notions de réputation, de menaces ou de représailles, qui peuvent apparaître quand on répète une situation du type « dilemme du prisonnier », ne sont donc effectives qu'à la condition que le jeu soit répété à l'infini (par les mêmes joueurs évidemment). Bien sûr, on pourrait admettre que le maire, même s'il risque de ne pas être réélu, est assez sensé pour comprendre que la commune, elle, vivra toujours et qu'il fera donc tout son possible jusqu' au bout pour favoriser l'intérêt de la région. Cherchons tout de même d'autres solutions...

\section{Méthodes d'incitation à la spécialisation}

Une autre méthode existe pour contrer une situation qui semble fatalement perdue d'avance comme le dilemme du prisonnier. Il s'agit de la «normalisation ». Kandori (1992a) explique qu'une normalisation permettrait d'obtenir une coopération entre les joueurs. Pour cela, il suffirait qu' une instance hiérarchiquement supérieure aux joueurs comme la région, l'État ou la Communauté européenne, «incite » les protagonistes à coopérer et punisse les non-coopérants. Elle pourrait, par exemple, (sévèrement) n'accorder les subventions que sous certaines conditions ou bien les fixer en fonction de la stratégie suivie par les auteurs du développement touristique ou encore (de manière plus diplomatique) calculer ses taux de crédits ou de subventions selon les choix effectués par les

récipiendaires, favorisant évidemment la stratégie « coopérer ». Les décideurs seraient alors incités à coopérer et donc, dans notre cas, à se spécialiser dans le domaine qui leur procure l'avantage comparatif le plus important.

Des formes « d'incitation » existent ; elles concernent en fait principalement la protection de l'environnement, mais certaines ont carrément pour but de favoriser le développement touristique par le biais d'une subvention, sous contrainte de respecter une orientation particulière, une ligne de conduite.

\section{Mesures « à caractère obligatoire » de protection et d'aménagement}

Des lois ont été votées dans le but de protéger l'environnement et donc les richesses naturelles d'une zone. Elles permettent d'empêcher une zone de mettre en œuvre des politiques qui iraient à l'encontre de ses propres intérêts et pourraient avoir des effets dévastateurs pour l'économie ou l'équilibre écologique de la zone.

- La Commission des unités touristiques nouvelles - Il s'agit d'une procédure gérée pour le compte de l'État qui s'applique aux communes non dotées de schémas directeurs désireuses, en matière d'aménagement en montagne, de créer une urbanisation, un équipement ou un aménagement touristique dans 
un site encore vierge de tout équipement, aménagement ou construction ; ou encore de créer une urbanisation, un équipement ou un aménagement touristique en discontinuité avec les urbanisations et les aménagements existants, mais entraînant une modification substantielle de l'économie locale, des paysages ou des équilibres naturels montagnards.

- La loi « montagne »- Loi qui permet notamment d'impulser une dynamique d'aménagement et de développement. Elle donne un réel pouvoir aux élus locaux pour maintenir les zones agricoles et mieux maîtriser le développement anarchique de l'urbanisme.

\section{- La protection et la mise en valeur du} littoral - Définissent une politique spécifique pour les communes riveraines des mers, des océans, des étangs salés et des lacs intérieurs d'une superficie supérieure à 1000 hectares. Elle s'applique également à certaines communes riveraines des estuaires et des deltas lorsqu'elles sont situées en aval de la limite de salure des eaux et participent aux équilibres économiques et écologiques littoraux.

- Le contrôle des stations balnéaires Les autorités communales déterminent la capacité d'accueil des espaces urbanisés afin de délimiter l'intensité du développement urbain de la commune. Cette capacité n'est pas calculée zone par zone, mais découle d'une approche globale qui porte sur une unité de territoire homogène.

Ces règles permettent un nouvel équilibre entre aménagement et protection, alliant ainsi développement économique et protection des écosystèmes littoraux. Elles constituent donc de nouvelles contraintes à intégrer dans les règles du jeu dont nous traitions précédemment. Un décideur de zone verte devra tenir compte du fait que l'une de ces lois pourrait l'empêcher d'implanter deux hôtels. Ces lois ne lui interdiront pas forcément l'implantation d'hôtels, mais elles l'empêcheront toutefois de les « placer» au mauvais endroit. Elles viendront donc favoriser les meilleures issues du jeu ; elles viendront favoriser par là même la spécialisation par zone et donc l'intérêt de la région tout entière.

\section{Interventions extérieures au jeu}

L'intervention d'acteurs hiérarchiquement supérieurs aux maires (ceux de la région ou du département notamment), vont l'aider à faire son choix en analysant le jeu (les jeux) de façon plus globale pour conseiller le décideur local dans son choix et faciliter l'organisation finale du territoire.

- L'aide des régions aux stations touristiques - Cette aide peut être octroyée par la région au Comité régional du tourisme (le C.R.T.) pour coordonner les efforts de promotion régionale, gérer et mettre en œuvre la politique touristique du Conseil régional.

- L'intervention départementale - « Le Schéma départemental de développement touristique permet de fixer une stratégie, de montrer que le développement ne se fait pas au coup par coup, que pour atteindre la cohérence il convient de définir des objectifs précis et de mobiliser les acteurs locaux. Le contenu du schéma intègre la réflexion stratégique sur l'aménagement, l'ensemble des activités touristiques et les choix politiques du département » (Vlès 1996). Le Comité départemental du tourisme est chargé du développement, voire de l'aménagement touristique départemental. Il est donc à l'écoute des stations touristiques pour pouvoir les conseiller dans leur projet d'équipement.

La décentralisation, en introduisant les schémas régionaux et départementaux du tourisme, a sans nul doute introduit un discours de la modernité dans la gestion du tourisme local. Les mises en cuvre des schémas, pour leur part, mettent en lumière les résistances locales au changement et le fonctionnement notabilier local traditionnel : les difficultés de l'inter-communalité, la propension au recours à l'investissement touristique dans le jeu électoral priment encore souvent (Vlès 1996).

\section{Les contrats}

Certaines mesures sont, comme nous l'avons déjà proposé intuitivement, basées sur des aides, des subventions. Elles n'ont donc pas le caractère obligatoire des précédentes, mais jouent un rôle « incitatif » tout aussi intéressant pour influencer (dans le bon sens) les stratégies retenues par nos décideurs. Ainsi, un système de « classification » des zones est proposé au maire. La règle est simple et peut se résumer ainsi : une subvention est accordée à la zone sous contrainte de respecter certains engagements relatifs à son aménagement. Il s'agit essentiellement des Contrats de station ; leur fonctionnement est également détaillé dans l'ouvrage de Vlès (1996).

- Les stations littorales - Elles bénéficient de contrats de revalorisation. Les programmes associés à cette appellation divergent selon les situations régionales.

- Les stations de montagne - Aide aux stations de moyenne importance, commercialisation de nouveaux produits et séjours. Deux conditions préalables sont demandées aux stations : la mise en place d'une structure unique d'animation et de commercialisation et la création d'une structure unique de gestion intercommunale.

- Les stations rurales - Hormis la valorisation touristique de quelques sites naturels ou culturels, les actions visent surtout la poursuite des actions les plus intéressantes qui s'étaient dégagées des contrats de pays d'accueil.

- La politique thermale - Les contrats de plan privilégient les objectifs économiques et commerciaux, les objectifs de modernisation des hébergements et des établissements existants et leur adaptation aux nouveaux produits.

\section{Les contrats de station}

Les politiques de contrat de pays d'accueil, de pôles, d'unités de séjour touristique visent à structurer l'espace touristique autour de pôles urbains suffisamment attractifs pour créer un effet de levier sur l'économie touristique. Elles visent ainsi à renforcer le capital « image » des stations, basé sur des thématiques affirmées et évocatrices de la «culture » locale, facilement repérables par le touriste. Cette politique permet de mobiliser l'ensemble des partenaires locaux autour d'une logique de développement. 
- Les contrats de pays d'accueil ou de pays côtier-Ces contrats sont destinés à améliorer les résultats de l'activité touristique par des interventions sur l'organisation des services et des entreprises touristiques, la modernisation de «l'existant» et la réalisation d'équipements d'accompagnement.

- Les contrats de station-vallée - Les objectifs sont identiques à ceux des pays d'accueil ; ils visent à établir ou à renforcer les liens professionnels entre les stations d'altitude et les bourgs de vallée. Ils permettent notamment le développement d'une vallée ou d'un massif autour d'une station de sports d'hiver.

- Les contrats de station de grand site touristique - Contrats destinés à conjuguer fréquentation touristique intense en site exceptionnel et sauvegarde de l'environnement.

- Les contrats de station touristique littorale ou de l'intérieur - Une station touristique classée ou non, littorale ou de l'intérieur, est une unité urbaine cohérente située à proximité d'équipements de liaison structurants (port, aéroport, grand axe routier), dotée d'équipements d'accueil, de services et de loisirs diversifiés de qualité, située dans un «pays » ou une micro-région et constituant, avec ces derniers, un ensemble touristique.

Ces contrats considèrent les stations comme des « entreprises de développement touristique ». Ils tentent de les organiser en leur donnant une image de marque, une gestion, une politique de commercialisation, en améliorant leur cadre de vie.

\section{Conclusion}

Tous les outils décrits permettent d'influencer le choix stratégique des décideurs. Ainsi, les contrats de stations touristiques littorales, de pays d'accueil ou côtiers et de stations de grand site permettent à certaines communes de recevoir des subventions si elles respectent les orientations établies au contrat. La commune de Ota-Porto en Corse (France), par exemple, obtenant l'appellation - et la subvention - de « station de grand site touristique », s'est engagée à entretenir son site classé UNESCO. Une zone peut donc, selon les spécificités qui lui sont propres, postuler pour la subvention dont les orientations correspondent au type de développement qui lui semble être le plus approprié. Elle reçoit de l'aide pour se spécialiser en fonction de ses atouts intrinsèques, ce qui facilite grandement les choix de développement et les prises de décisions des responsables locaux.

Denis Serra est enseignant en économie au lycée de Porto-Vecchio (Corse, France). Il a été adjoint à la rédaction de la revue Téoros, stagiaire postdoctoral et auxiliaire d'enseignement à l'Université $d u$ Québec à Montréal.

\section{Notes}

1 Une zone de « luxe » $(\mathrm{L})$, d'aspect relativement confortable et faiblement sensible, dispose d'attractions touristiques, d'activités commerciales notables et d'une capacité d'accueil relativement importante; de plus, elle est facilement accessible. D'autre part, elle ne dispose ni d'un patrimoine historique ni d'une faune ou d'une flore remarquable

2 Une zone « verte » $(\mathrm{V})$, d'aspect relativement sensible et faiblement confortable, dispose d'une faune et d'une flore jusqu' $\mathrm{i}$ ci entretenues et protégées. Elle est d'un aspect pittoresque certain, pas forcément très accessible, mais pas isolée non plus. Elle n'est pas excessivement développée commercialement : on y trouve quelques commerces, mais pas de luxe (plutôt pizzeria que restaurant avec étoiles, plutôt cinéma de plein air que casino). Elle ne dispose ni d'une grande capacité d'accueil, ni d'une forte attraction touristique, si ce n'est de son aspect proche de la nature.

3 Dans notre étude, nous appelons flux monétaire la quantité totale de monnaie dépensée par un visiteur dans sa zone de résidence. Cela signifie que ce flux sera « attribué » à l'établissement d'hébergement qu'il aura choisi. Ainsi, le flux monétaire sera constitué non seulement des frais d'hébergement, mais aussi des dépenses effectuées par le client sur toute sa zone d'accueil (commerces, restaurants, discothèques, souvenirs achetés chez un artisan). Les dépenses effectuées sur une zone voisine (du lieu d'hébergement) ne seront pas prises en compte dans le calcul du flux monétaire, pas plus que les frais de transport (aller comme retour) relatifs à l'acheminement du voyageur jusqu'à sa zone, puisque nous estimons, dans cette recherche, que quelle que soit la stratégie de développement choisie par le décideur d'une zone, elle n'aura que très peu d'influence (voire pas du tout) sur le choix du consommateur quant au moyen de transport qu'il retiendra pour se rendre à son lieu de villégiature.

\section{Bibliogaphie}

Axelrod, Robert (1984), The Evolution of Cooperation, Basic Book.

Attali, Jacques (1972), Les modèles politiques, Presse Universitaire Française.

Bouzitat, Jean (1965), Présentation synthétique de la théorie des jeux, Bureau universitaire de recherche opérationnelle.

Gabszewick, Jean (1994), La concurrence imparfaite, La Découverte.

Guerrien, Bernard (1993), La théorie des jeux, 2 e éd., Paris, Economica.

Kandori, M. (1992a), « Social Norms and Community Enforcement », Review of Economic Studies.

Kandori, M. (1992b), Repeated Games Played by Overlapping Generations of Players, R.E.S.

Serra, Denis (2001), « Tourisme identitaire et spécialisation infra-régionale dans les petites économies isolées », Téoros « Organisations touristiques en mutation », 19 (3).

Serra, Denis (2000), « Tourisme et développement régional : proposition d'une stratégie de spécialisation infra-régionale adaptée aux spécificités des petites économies isolées », Thèse de Doctorat ès sciences économiques de l'Université de Corse.

Serra, Denis (1998), « Coopérer par la spécialisation : pour une organisation du tourisme Méditerranéen », dans Cooperation and Development in the Mediterranean, Colloque international Première rencontre des économistes méditerranéens, Split, Croatie.

Serra, Denis (1997), « Réflexion sur l'organisation du tourisme insulaire fondée sur l'analyse de l'offre et de la demande », dans Économie des Régions Méditerranéennes et Développement Durable, Colloque international, Arles, France.

Smith, Adam (1776), Recherche sur la nature et les causes de la richesse des nations.

Vlès, Vincent (1996), « Les stations touristiques », Paris, Economica.

Vlès, Vincent (2001), Service public touristique local et aménagement du territoire, Paris, L'Harmattan. 